Pacific Journal of Mathematics

CROSSED PRODUCT AND HEREDITARY ORDERS

DOW ARD CUFF AND ALFRED RHENHOLD WeISs 


\title{
CROSSED PRODUCT AND HEREDITARY ORDERS
}

\author{
Gerald H. Cliff and Alfred R. Weiss
}

Let $\Lambda$ be the crossed product order $\left(O_{L} / O_{K}, G, \rho\right)$ where $L / K$ is a finite Galois extension of local fields with Galois group $G$, and $\rho$ is a factor set with values in $O_{L}^{*}$. Let $\Lambda_{0}=\Lambda$, and let $\Lambda_{i+1}$ be the left order $O_{l}\left(\operatorname{rad} \Lambda_{i}\right)$ of $\operatorname{rad} \Lambda_{l}$. The chain of orders $\Lambda_{0}, \Lambda_{1}, \ldots, \Lambda_{s}$ ends with a hereditary order $\Lambda_{s}$. We prove that $\Lambda_{s}$ is the unique minimal hereditary order in $A=K \Lambda$ containing $\Lambda$, that $\Lambda_{s}$ has $e / m$ simple modules, each of dimension $f$ over the residue class field $\bar{K}$ of $O_{K}$, and that $s=d-$ $(e-1)$. Here $d, e, f$ are the different exponent, ramification index, and inertial degree of $L / K$, and $m$ is the Schur index of $A$.

1. Introduction. Let $O_{K}$ be a complete discrete valuation ring having field of fractions $K$ and finite residue class field $\bar{K}$. Let $L$ be a finite Galois extension of $K$, with Galois group $G$, and let $O_{L}$ be the valuation ring in $L$. Let $\rho$ be a factor set on $G \times G$ with values in the units of $O_{L}$. We are interested in the crossed product order $\Lambda=\left(O_{L} / O_{K}, G, \rho\right)$ contained in the simple algebra $A=(L / K, G, \rho)$. If $\rho$ is trivial, AuslanderGoldman [1] showed that $\Lambda$ is a maximal order in $A$ if and only if $L / K$ is unramified, and Auslander-Rim [2] showed that $\Lambda$ is hereditary if and only if $L / K$ is tamely ramified. Williamson [8] extended the AuslanderRim result to the case that $\rho$ is any factor set. We are interested in the wild case. Benz-Zassenhaus [3] showed that $\Lambda$ is contained in a unique minimal hereditary order in $A$.

We set $\Lambda_{0}=\Lambda$, and define inductively

$$
\Lambda_{j+1}=\left\{x \in A: x \operatorname{rad} \Lambda_{j} \subseteq \operatorname{rad} \Lambda_{j}\right\}=O_{l}\left(\operatorname{rad} \Lambda_{j}\right) .
$$

Then we have the sequence of orders

$$
\Lambda_{0} \varsubsetneqq \Lambda_{1} \varsubsetneqq \Lambda_{2} \varsubsetneqq \cdots \varsubsetneqq \Lambda_{s}=\Lambda_{s+1}
$$

for some integer $s$. Since $\Lambda_{s}=O_{l}\left(\operatorname{rad} \Lambda_{s}\right)$, it follows that $\Lambda_{s}$ is hereditary $([6,39.11,39.14])$. From the theory of hereditary orders (see $[6,39.14]) \Lambda_{s}$ may be described as follows: if $A \cong M_{n}(D)$, the ring of $n \times n$ matrices over a division ring $D$, and if $\Delta$ is the unique maximal order in $D$, then $\Lambda_{s}$ is the set of block matrices, with entries in $\Delta$, where there are $r$ diagonal blocks of size $n_{i} \times n_{i}$, and blocks above the diagonal have 
entries in $\operatorname{rad} \Delta$. The positive integer $r$ is called the type number of $\Lambda_{s}$, and is also equal to the number of simple $\Lambda_{s}$-modules. Our main result is the following.

Theorem. (1) $\Lambda_{s}$ is the unique minimal hereditary $O_{K^{-}}$order in $A$ containing $\Lambda$.

(2) $\operatorname{rad} \Lambda_{s}=P_{L} \Lambda_{s}$, where $P_{L}$ denotes the maximal ideal of $O_{L}$.

(3) $r=e / m$, where $e$ is the ramification index of $L / K$ and $m$ is the Schur index of $A$.

(4) $n_{1}=n_{2}=\cdots=n_{r}=f$, the inertial degree of $L / K$.

(5) $s=d-(e-1)$, where $d$ is the exponent $P_{L}^{d}=\mathscr{D}$ of the different of $L / K$.

We prove this by first considering the split case (when $\rho=1$ ), and then taking an unramified extension $K^{\prime}$ of $K$ which splits $A$, and considering $A \otimes_{K} K^{\prime}$ which is a crossed product $\left(L^{\prime} / K^{\prime}, G, 1\right)$, where $L^{\prime}=L \otimes_{K} K^{\prime}$. Then $L^{\prime}$ is not in general a field, but a Galois algebra over $K^{\prime}$, and we find it convenient to prove the Theorem when $L$ is a Galois algebra over $K$ to begin with; we take $O_{L}$ to be the integral closure of $O_{K}$ in $L$, we replace $P_{L}$ by $\operatorname{rad} O_{L}$, and we give suitable definitions of $d, e$, and $f$ in $\S 2$. We deal with the split case in $\S 3$, and the general case in $\S 4$. We find generators for the hereditary order $\Lambda_{s}$ in $\$ 5$, in the totally ramified split case. In $\S 6$ we show how our results yield those of Auslander-Goldman-Rim-Williamson, as well as some others.

We cite Reiner [6] as a general reference.

2. Galois algebras. Let $L$ be a commutative Galois algebra over $K$, with finite Galois group $G$, by which we mean that $L$ is a commutative separable $K$-algebra with $G$ a group of automorphisms of $L$ fixing $K$ such that the fixed subalgebra $L^{G}=K$ and $|G|=\operatorname{dim}_{K} L$. Let $O_{L}$ be the integral closure of $O_{K}$ in $L$. Let $E$ denote the set of primitive idempotents of $L$. Then for $\varepsilon \in E$, the integral closure $O_{L \varepsilon}$ of $O_{K}$ in the field $L \varepsilon$ is a complete discrete valuation ring, and $O_{L \varepsilon}=O_{L} \varepsilon$. Since $L^{G}=K, G$ acts transitively on $E$.

LEMMA 2.1. Let $I$ be a non-zero $O_{L^{-}}$submodule of $L$ which is $G$-invariant. Then $I=\left(\operatorname{rad} O_{L}\right)^{i}$ for some integer $i$.

Proof. For any primitive idempotent $\varepsilon$ of $L, I \varepsilon$ is a non-zero $O_{L \varepsilon}$-submodule of $L \varepsilon$, and therefore $I \varepsilon=\left(\operatorname{rad} O_{L_{\varepsilon}}\right)^{i_{\varepsilon}}$ for some $i_{\varepsilon} \in \mathbf{Z}$, since $O_{L \varepsilon}$ is a discrete valuation ring. Because $G$ acts transitively on $E$ 
and $I$ is $G$-invariant, it follows that $i_{\varepsilon}=i$ is independent of $\varepsilon$. Then

$$
I=\sum_{\varepsilon \in E} I \varepsilon=\sum_{\varepsilon \in E}\left(\operatorname{rad} O_{L \varepsilon}\right)^{i}=\sum_{\varepsilon \in E}\left(\operatorname{rad} O_{L}\right)^{i} \varepsilon=\left(\operatorname{rad} O_{L}\right)^{i}
$$

as desired.

First, let $I=P_{K} O_{L}$. Then $P_{K} O_{L}=\left(\operatorname{rad} O_{L}\right)^{e}$ for some integer $e$, and we call $e$ the ramification index of $L / K$.

Next, let $\operatorname{tr}_{L / K}: L \rightarrow K$ be the trace map, and let

$$
\tilde{O}_{L}=\left\{x \in L: \operatorname{tr}_{L / K}\left(x O_{L}\right) \subseteq O_{K}\right\}
$$

be the complementary module to $O_{L}$ under the trace. Since

$$
\operatorname{tr}_{L / K}(x)=\sum_{g \in G} g(x), \quad x \in L,
$$

it follows that $\tilde{O}_{L}$ is a $G$-invariant $O_{L}$-submodule of $L$, so $\tilde{O}_{L}=\left(\operatorname{rad} O_{L}\right)^{-d}$ for some integer $d$. We call $d$ the different exponent of $L / K$ (and $\left(\operatorname{rad} O_{L}\right)^{d}$ the different $\mathscr{D}_{L / K}$ of $\left.L / K\right)$.

Define the inertial degree $f$ of $L / K$ to be $\operatorname{dim}_{\bar{K}}\left(O_{L} / \operatorname{rad} O_{L}\right)$.

Let $\rho: G \times G \rightarrow O_{L}^{*}$ be a factor set on $G$ with values in the units of $O_{L}$. The crossed product algebra $A=(L / K, G, \rho)$ is the free left $L$-module with basis $u_{g}, g \in G$, with multiplication given by

$$
x u_{g} \cdot y u_{h}=x g(y) \rho(g, h) u_{g h}, \quad x, y \in L, g, h \in G .
$$

The order $\Lambda=\left(O_{L} / O_{K}, G, \rho\right)$ is the $O_{L}$-submodule of $A$ spanned by $u_{g}$, $g \in G$. We assume that $\rho(g, 1)=\rho(1, g)=1$, so that $O_{L}$ may be identified inside $\Lambda$ as $\left\{x u_{1}: x \in O_{L}\right\}$.

LEMMA 2.2. (1) $L$ has a normal $K$-basis with respect to $G$.

(2) $A$ is a central simple $K$-algebra, and $A$ is isomorphic to a full matrix ring over $K$ if and only if the class of $\rho$ in $H^{2}\left(G, L^{*}\right)$ is 1 .

(3) The reduced trace $\operatorname{trd}: A \rightarrow K$ is given by

$$
\operatorname{trd}\left(\sum_{g \in G} a_{g} u_{g}\right)=\operatorname{tr}_{L / K}\left(a_{1}\right) .
$$

Proof. These results are well known if $L$ is a field, and the proofs are essentially the same if $L$ is a Galois algebra. We omit the details.

3. The split case. In this section we assume that $L / K$ is a Galois algebra, and we prove the theorem in the case that $\rho=1$, with $P_{L}$ replaced by $\operatorname{rad} O_{L}$, and with $d, e, f$ defined as in $\S 2$. Since $\rho=1$, then 
$A \cong M_{n}(K), n=|G|$. Let $V$ be a simple $A$-module. The structure theory for hereditary orders $([\mathbf{6}, 39.18])$ provides a $\Lambda_{s}$-submodule $M$ contained in $V$ with the following properties:

(a) $r$ is the unique positive integer such that $\left(\operatorname{rad} \Lambda_{s}\right)^{r} M=P_{K} M$, (since $\operatorname{End}_{A}(V)=K$ ).

(b) $\Lambda_{s}=\left\{x \in A: x\left(\operatorname{rad} \Lambda_{s}\right)^{i} M \subseteq\left(\operatorname{rad} \Lambda_{s}\right)^{l} M, 0 \leq i<r\right\}$.

(c) $\operatorname{rad} \Lambda_{\mathrm{s}}=\left\{\mathrm{x} \in \mathrm{A}: \mathrm{x}\left(\operatorname{rad} \Lambda_{\mathrm{s}}\right)^{\mathrm{i}} \mathrm{M} \subseteq\left(\operatorname{rad} \Lambda_{\mathrm{s}}\right)^{i+1} M, 0 \leq i<r\right\}$.

(d) $\left(\operatorname{rad} \Lambda_{s}\right)^{t-1} M /\left(\operatorname{rad} \Lambda_{s}\right)^{i} M, 1 \leq i \leq r$, are a full set of simple $\Lambda_{s}$ modules.

(e) $n_{l}=\operatorname{dim}_{\bar{K}}\left(\operatorname{rad} \Lambda_{s}\right)^{i-1} M /\left(\operatorname{rad} \Lambda_{s}\right)^{l} M, 1 \leq i \leq r$.

The algebra $A$ acts on $L$, via

$$
\left(\sum x_{g} u_{g}\right) \cdot y=\sum x_{g} g(y), \quad \sum x_{g} u_{g} \in A, y \in L,
$$

and acts irreducibly on $L$, so we may take $L$ to be $V$. The non-zero $\Lambda$-submodules of $L$ are $O_{L}$-submodules of $L$ which are $G$-stable, so they are precisely $\left(\operatorname{rad} O_{L}\right)^{i}, i \in \mathbf{Z}$, by Lemma 2.1. We denote the $\Lambda$-module $\left(\operatorname{rad} O_{L}\right)^{i}$ by $M_{i}$.

LEMMA 3.1. For each integer $j \geq 0$,

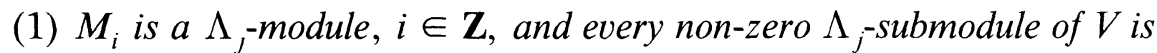
$M_{i}$ for some $i$.

(2) $\left(\operatorname{rad} \Lambda_{j}\right) M_{l}=M_{l+1}$.

Proof. If (1) holds for some $j$, then $\left(\operatorname{rad} \Lambda_{j}\right) M_{\imath} \varsubsetneqq M_{\imath}$, by Nakayama's Lemma, so $\left(\operatorname{rad} \Lambda_{j}\right) M_{i} \subseteq M_{i+1}$, since $M_{i+1}$ is the unique maximal $\Lambda_{j}$-submodule of $M_{\imath}$. But $\operatorname{rad} O_{L} \subseteq \operatorname{rad} \Lambda_{\jmath}$, since $\left(\operatorname{rad} O_{L}\right) M_{\imath} \subsetneq M_{\imath}$ for each $i$, and $\left(\operatorname{rad} O_{L}\right) M_{\imath}=M_{\imath+1}$, so $\left(\operatorname{rad} \Lambda_{J}\right) M_{\imath}=M_{\imath+1}$, proving (2). For (1), we use induction on $j$, having noted that it holds for $\Lambda_{0}$. Then for $j+1$,

$$
\begin{aligned}
\Lambda_{J+1} M_{l} & =\Lambda_{J+1}\left(\operatorname{rad} \Lambda_{j}\right) M_{l-1} \quad(\text { by }(2) \text { for } j) \\
& \subseteq\left(\operatorname{rad} \Lambda_{j}\right) M_{l-1} \quad\left(\text { by definition of } \Lambda_{i+1}\right) \\
& =M_{l}
\end{aligned}
$$

so $M_{\imath}$ is a $\Lambda_{j+1}$-module, $i \in \mathbf{Z}$. Since any $\Lambda_{j+1}$-module is also a $\Lambda$-module, the proof is complete.

LEMMA 3.2. (1) $\Lambda_{s}=\left\{x \in A: x M_{t} \subseteq M_{\imath}, i \in \mathbf{Z}\right\}$.

(2) $\operatorname{rad} \Lambda_{s}=\left\{x \in A: x M_{i} \subseteq M_{i+1}, i \in \mathbf{Z}\right\}$.

(3) $\operatorname{rad} \Lambda_{s}=\left(\operatorname{rad} O_{L}\right) \Lambda_{s}=\Lambda_{s}\left(\operatorname{rad} O_{L}\right)$. 
Proof. The structure of $\Lambda_{s}$ is given in terms of a $\Lambda_{s}$-submodule $M$ contained in $V$. From Lemma 3.1, any $\Lambda_{s}$-submodule of $V$ must be $M_{k}$ for some integer $k$. We have, from (b) and Lemma 3.1,

$$
\Lambda_{s}=\left\{x \in A: x M_{k+l} \subseteq M_{k+i}, 0 \leq i<r\right\} .
$$

From (a), $M_{k+r}=\left(\operatorname{rad} \Lambda_{s}\right)^{r} M_{k}=P_{K} M_{k}$, and since $P_{K}$ is a principal ideal of $O_{K}$, then $M_{k+r} \cong M_{k}$ as $\Lambda_{s}$-modules. Then for $i \in \mathbf{Z}$,

$$
\left(\operatorname{rad} \Lambda_{s}\right)^{l} M_{k+r}=M_{i+k+r} \cong\left(\operatorname{rad} \Lambda_{s}\right)^{l} M_{k}=M_{l+k}
$$

so $M_{\imath+r} \cong M_{i}$ as $\Lambda_{s}$-modules, $i \in \mathbf{Z}$. Thus

$$
\Lambda_{s}=\left\{x \in A: x M_{i} \subseteq M_{\imath}, i \in \mathbf{Z}\right\},
$$

proving (1), and (2) follows from (1). Since $\operatorname{rad} O_{L} \subseteq \operatorname{rad} \Lambda_{s}$ and $\left(\operatorname{rad} O_{L}\right) M_{i}=M_{i+1}=\left(\operatorname{rad} \Lambda_{s}\right) M_{i}, i \in \mathbf{Z},(3)$ follows from (2).

Parts (1)-(4) of the Theorem are now straightforward in this case. If $\Gamma$ is a hereditary order in $A$ containing $\Lambda$, then applying the structure theory to $\Gamma$, there is a $\Gamma$-submodule $M$ of $V$ such that

$$
\Gamma=\left\{x \in A: x(\operatorname{rad} \Gamma)^{l} M \subseteq(\operatorname{rad} \Gamma)^{l} M, 1 \leq i \leq \text { type number of } \Gamma\right\} .
$$

Since $\Lambda \subseteq \Gamma, M$ is a $\Lambda$-module, so $M=M_{j}$ for some integer $j$. Also, since $\left(\operatorname{rad} O_{L}\right) M_{i} \subsetneq M_{i}, i \in \mathbf{Z}$, then $\operatorname{rad} O_{L} \subseteq \operatorname{rad} \Gamma$, and then $(\operatorname{rad} \Gamma)^{i} M$, $=M_{J+l}, i \in \mathbf{Z}$. It follows from Lemma 3.2 that $\Lambda_{s} \subseteq \Gamma$, proving (1) of the theorem. Part (2) is contained in Lemma 3.2. For (3), we know from (a) that $r$ is the integer such that $\left(\operatorname{rad} \Lambda_{s}\right)^{r} M_{k}=P_{K} M_{k}$. But

$$
P_{K} M_{k}=P_{K} O_{L} M_{k}=\left(\operatorname{rad} O_{L}\right)^{e} M_{k}=M_{k+e}
$$

so $r=e$. (Note that $m=1$ here.) For (4),

$$
\begin{aligned}
& \left(\operatorname{rad} \Lambda_{s}\right)^{t-1} M_{k} /\left(\operatorname{rad} \Lambda_{s}\right)^{t} M_{k} \\
& \quad=M_{k+i-1} / M_{k+l}=\left(\operatorname{rad} O_{L}\right)^{k+\imath-1} /\left(\operatorname{rad} O_{L}\right)^{k+i}
\end{aligned}
$$

and as $\bar{K}$-modules $\left(\operatorname{rad} O_{L}\right)^{k+1-1} /\left(\operatorname{rad} O_{L}\right)^{k+i} \cong O_{L} / \operatorname{rad} O_{L}$ so

$$
n_{l}=\operatorname{dim}_{\bar{K}} O_{L} / \operatorname{rad} O_{L}=f, 1 \leq i \leq r .
$$

In order to prove (5), we use the following result.

Lemma 3.3. Suppose that $a$ is an integer $\geq 0$ such that $\left(\operatorname{rad} \Lambda_{s}\right)^{a}$ is the largest left $\Lambda_{s}$-ideal contained in $\Lambda$. Then $s=a$.

Proof. If $a=0$, then $\Lambda_{s} \subseteq \Lambda$, so $\Lambda_{s}=\Lambda$, and $s=0$. Assuming that $a>0$, we show that $\left(\operatorname{rad} \Lambda_{s}\right)^{a-1}$ is the largest left $\Lambda_{s}$-ideal contained in $\Lambda_{1}$. First,

$$
\left(\operatorname{rad} \Lambda_{s}\right)^{a-1} \operatorname{rad} \Lambda \subseteq\left(\operatorname{rad} \Lambda_{s}\right)^{a-1} \operatorname{rad} \Lambda_{s}=\left(\operatorname{rad} \Lambda_{s}\right)^{a} .
$$


Now $\left(\operatorname{rad} \Lambda_{s}\right)^{a} \subseteq \Lambda$ by hypothesis, and $\operatorname{rad} \Lambda_{s} \cap \Lambda \subseteq \operatorname{rad} \Lambda$, by Lemma 3.2. Thus $\left(\operatorname{rad} \Lambda_{s}\right)^{a} \subseteq \operatorname{rad} \Lambda$. Then $\left(\operatorname{rad} \Lambda_{s}\right)^{a-1}(\operatorname{rad} \Lambda) \subseteq \operatorname{rad} \Lambda$, so $\left(\operatorname{rad} \Lambda_{s}\right)^{a-1} \subseteq \Lambda_{1}$.

Next, if $L$ is a left $\Lambda_{s}$-ideal contained in $\Lambda_{1}$, then $L \operatorname{rad} \Lambda \subseteq \operatorname{rad} \Lambda$, so $L \operatorname{rad} \Lambda \subseteq\left(\operatorname{rad} \Lambda_{s}\right)^{a}$. Then

$$
L \operatorname{rad} \Lambda_{s}=L(\operatorname{rad} \Lambda) \Lambda_{s} \subseteq\left(\operatorname{rad} \Lambda_{s}\right)^{a} .
$$

Since $\operatorname{rad} \Lambda_{s}$ is invertible, $L \subseteq\left(\operatorname{rad} \Lambda_{s}\right)^{a-1}$ as desired.

Now by induction, the length of the chain $\Lambda_{1} \subseteq \Lambda_{2} \subseteq \cdots \subseteq \Lambda_{s}$ is $a-1$, so $s=a$, and the proof is complete.

Let trd : $A \rightarrow K$ be the reduced trace, and for an $O_{K}$-submodule $L$ of $A$ with $K L=A$, let

$$
\tilde{L}=\left\{x \in A: \operatorname{trd}(x L) \subseteq O_{K}\right\}
$$

be the complementary module.

LEMMA 3.4. Let $\Gamma$ be any hereditary $O_{K}$ order contained in the split simple algebra $A=M_{n}(K)$. Then

$$
\tilde{\Gamma}=P_{K}^{-1} \operatorname{rad} \Gamma \text {. }
$$

Proof. Suppose that $\Gamma$ has type number $r$, invariants $n_{1}, \ldots, n_{r}$, and $\Gamma$ consists of block matrices as mentioned in section 1 . Let $\pi_{K}$ be a prime element of $O_{K}$. For integers $i, j, 1 \leq i, j \leq n$, let $Y_{i j}$ denote the matrix whose $i, j$-entry is $\pi_{K}$ if the $i, j$-position is above the diagonal of blocks of $\Gamma$, or 1 otherwise, and all of whose other entries are 0 (so $Y_{i j} \in \Gamma$.) Let $y_{i j}$ denote the non-zero entry of $Y_{i j}$. Let $X=\left(x_{i j}\right)$ be any element of $A$. Then $X Y_{i j}$ has at most one non-zero entry on the main diagonal, namely $x_{i j} y_{j i}$. We have $\operatorname{trd}\left(X Y_{i j}\right)=$ trace of matrix $X Y_{i j}=x_{i j} y_{j i}$. Then $X \in \tilde{\Gamma}$ $\Leftrightarrow x_{i j} y_{j i} \in O_{K}$, all $i, j \Leftrightarrow$ when $X$ is partitioned according to the block partition induced by $\Gamma$, the entries below the diagonal of blocks are in $P_{K}^{-1}$, and the other entries are in $O_{K}$. But such matrices are precisely those in $P_{K}^{-1} \operatorname{rad} \Gamma$. Since the $Y_{i j}$ give a free basis for $\Gamma$ over $O_{K}$, the result follows.

LEMMA 3.5. Let $w=d-(e-1)$. Then $\left(\operatorname{rad} \Lambda_{s}\right)^{w}$ is the largest left $\Lambda_{s}$-ideal contained in $\Lambda$.

Proof. From Lemma 3.2, we have $\operatorname{rad} \Lambda_{s}=\left(\operatorname{rad} O_{L}\right) \Lambda_{s}$, so $\left(\operatorname{rad} \Lambda_{s}\right)^{w}$ $=\left(\operatorname{rad} O_{L}\right)^{d-(e-1)} \Lambda_{s}$. From Lemma 3.4

$$
\tilde{\Lambda}_{s}=P_{K}^{-1} \operatorname{rad} \Lambda_{s}=\left(\operatorname{rad} O_{L}\right)^{-e}\left(\operatorname{rad} O_{L}\right) \Lambda_{s}=\left(\operatorname{rad} O_{L}\right)^{-e+1} \Lambda_{s},
$$


so

$$
\left(\operatorname{rad} \Lambda_{s}\right)^{w}=\left(\operatorname{rad} O_{L}\right)^{d} \tilde{\Lambda}_{s}=\left(\left(\operatorname{rad} O_{L}\right)^{-d} \Lambda_{s}\right)^{\sim} .
$$

From Lemma 2.2, $\operatorname{trd}\left(\sum x_{g} u_{g}\right)=\operatorname{tr}_{L / K}\left(x_{1}\right)$, so

$$
\begin{gathered}
\tilde{\Lambda}=\mathscr{D}^{-1} \Lambda=\left(\operatorname{rad} O_{L}\right)^{-d} \Lambda \subseteq\left(\operatorname{rad} O_{L}\right)^{-d} \Lambda_{s}, \\
\left(\operatorname{rad} \Lambda_{s}\right)^{w}=\left(\left(\operatorname{rad} O_{L}\right)^{-d} \Lambda_{s}\right)^{\sim} \subseteq \tilde{\Lambda}=\Lambda,
\end{gathered}
$$

so $\left(\operatorname{rad} \Lambda_{s}\right)^{w}$ is contained in $\Lambda$. If $L$ is any other left $\Lambda_{s}$-ideal contained in $\Lambda$, then $\tilde{L}$ is a right $\Lambda_{s}$-module containing $\tilde{\Lambda}$, so

$$
\begin{aligned}
& \tilde{L} \supseteq \tilde{\Lambda} \Lambda_{s}=\mathscr{D}^{-1} \Lambda_{s}=\left(\operatorname{rad} O_{L}\right)^{-d} \Lambda_{s}, \\
& L=\tilde{\tilde{L}} \subseteq\left(\left(\operatorname{rad} O_{L}\right)^{-d} \Lambda_{s}\right)^{\sim}=\left(\operatorname{rad} \Lambda_{s}\right)^{w},
\end{aligned}
$$

completing the proof.

Now (5) of the Theorem follows from Lemmas 3.3 and 3.5.

4. The general case. In this section we continue with the assumption that $L / K$ is a Galois algebra, and we prove the Theorem in the case that $\rho$ is any factor set with values in $O_{L}^{*}$. Since $\bar{K}$ is finite, there is an unramified field extension $K^{\prime}$ of $K$ such that the algebra $A^{\prime}=A \otimes_{K} K^{\prime}$ splits ([7, Prop. 2, p. 191].) Let $O^{\prime}$ be the integral closure of $O_{K}$ in $K^{\prime}$, and let $\Lambda^{\prime}=\Lambda \otimes_{O_{K}} O^{\prime}$.

LeMma 4.1. If $\Gamma$ is an $O_{K}$-order, then

$$
\operatorname{rad}\left(\Gamma \otimes_{O_{K}} O^{\prime}\right)=(\operatorname{rad} \Gamma) \otimes_{O_{K}} O^{\prime} .
$$

Proof. Denote $O_{K}$ by $O$, and $P_{K}$ by $P$. Clearly

$$
(\operatorname{rad} \Gamma) \otimes_{O} O^{\prime} \subseteq \operatorname{rad}\left(\Gamma \otimes_{O} O^{\prime}\right) .
$$

For the reverse inclusion, we have

$$
\left(\Gamma \otimes_{O} O^{\prime}\right) /(\operatorname{rad} \Gamma) \otimes_{O} O^{\prime} \cong(\Gamma / \operatorname{rad} \Gamma) \otimes_{O} O^{\prime} .
$$

Since $P \subseteq \operatorname{rad} \Gamma$, then $\Gamma / \operatorname{rad} \Gamma$ is an $O / P$-module, and

$$
(\Gamma / \operatorname{rad} \Gamma) \otimes_{O} O^{\prime} \cong(\Gamma / \operatorname{rad} \Gamma) \otimes_{O / P}\left(O^{\prime} / P O^{\prime}\right) .
$$

Since $K^{\prime} / K$ is unramified, then $O^{\prime} / P O^{\prime}$ is field, which is separable over $\bar{K}$ since $\bar{K}$ is finite. Then the semi-simple $O / P$-algebra $\Gamma / \mathrm{rad} \Gamma$ remains semi-simple after tensoring with $O^{\prime} / P O^{\prime}$, so $\Gamma \otimes_{O} O^{\prime} /(\operatorname{rad} \Gamma) \otimes_{O} O^{\prime}$ is semi-simple, and the result follows. 
We let $G$ act on $L^{\prime}=L \otimes_{K} K^{\prime}$ by

$$
g(x \otimes y)=g(x) \otimes y, \quad x \in L, y \in K^{\prime}, g \in G .
$$

Then $L^{\prime}$ is a Galois algebra over $K^{\prime}$ with Galois group $G$. We have $O_{L^{\prime}}=O_{L} \otimes_{O_{K}} O^{\prime}$, and

$$
\Lambda^{\prime}=\Lambda \otimes_{O_{K}} O^{\prime}=\left(O_{L^{\prime}} / O^{\prime}, G, \rho\right) .
$$

Let us show that in going from $L / K$ to $L^{\prime} / K^{\prime}$, the numbers $d, e, f$ are unchanged.

Applying Lemma 4.1 to the $O_{K}$-order $O_{L}$, we have $\operatorname{rad} O_{L^{\prime}}=$ $\left(\operatorname{rad} O_{L}\right) \otimes_{O_{K}} O^{\prime}$. Since the maximal ideal $P^{\prime}$ of $O^{\prime}$ is $P_{K} O^{\prime}$, then

$$
P^{\prime} O_{L^{\prime}}=\left(P_{K} O_{L}\right) \otimes_{O_{K}} O^{\prime}=\left(\operatorname{rad} O_{L}\right)^{e} \otimes_{O_{K}} O^{\prime}=\left(\operatorname{rad} O_{L^{\prime}}\right)^{e}
$$

so the ramification index of $L^{\prime} / K^{\prime}$ is still $e$. Similarly,

$$
\operatorname{dim}_{\bar{K}^{\prime}}\left(O_{L^{\prime}} / \operatorname{rad} O_{L^{\prime}}\right)=\operatorname{dim}_{\bar{K}}\left(O_{L} / \operatorname{rad} O_{L}\right)=f .
$$

For the different exponent of $L^{\prime} / K^{\prime}$, since

$$
\operatorname{tr}_{L^{\prime} / K^{\prime}}(x \otimes y)=\operatorname{tr}_{L / K}(x) \otimes y, \quad x \in L, y \in K^{\prime},
$$

then clearly $\tilde{O}_{L} \otimes_{O_{K}} O^{\prime} \subseteq \tilde{O}_{L^{\prime}} ;$ since $\tilde{O}_{L}=\left(\operatorname{rad} O_{L}\right)^{-d}$, and $\operatorname{rad} O_{L^{\prime}}=$ $\left(\operatorname{rad} O_{L}\right) \otimes_{O_{K}} O^{\prime}$, then $\left(\operatorname{rad} O_{L^{\prime}}\right)^{-d} \subseteq \tilde{O}_{L^{\prime}}$. If $\left(\operatorname{rad} O_{L^{\prime}}\right)^{-d-1} \subseteq \tilde{O}_{L^{\prime}}$, then $\left(\operatorname{rad} O_{L}\right)^{-d-1} \subseteq \tilde{O}_{L}$, which is not so. Therefore $\tilde{O}_{L^{\prime}}=\left(\operatorname{rad} O_{L^{\prime}}\right)^{-d}$.

LEMMA 4.2. If $\Gamma$ is an $O_{K^{-}}$order contained in a semi-simple algebra A, then

$$
O_{l}(\operatorname{rad} \Gamma) \otimes_{O_{K}} O^{\prime}=O_{l}\left(\operatorname{rad}\left(\Gamma \otimes_{O_{K}} O^{\prime}\right)\right) .
$$

Proof. It is clear that the left side is contained in the right. There is an isomorphism

$$
\phi: O_{l}(\operatorname{rad} \Gamma) \rightarrow \operatorname{Hom}_{\Gamma}(\operatorname{rad} \Gamma, \operatorname{rad} \Gamma),
$$

where $\operatorname{rad} \Gamma$ is considered as a right $\Gamma$-module. Similarly, there is an isomorphism

$$
\psi: O_{l}\left(\operatorname{rad} \Gamma^{\prime}\right) \rightarrow \operatorname{Hom}_{\Gamma^{\prime}}\left(\operatorname{rad} \Gamma^{\prime}, \operatorname{rad} \Gamma^{\prime}\right),
$$

where $\Gamma^{\prime}=\Gamma \otimes_{\mathrm{O}} \mathrm{O}^{\prime}$. Since $\Gamma$ is noetherian, then $\operatorname{rad} \Gamma$ is finitely presented over $\Gamma$, so from $[6,2.37]$ we have an isomorphism

$$
\begin{aligned}
\sigma: \operatorname{Hom}_{\Gamma}(\operatorname{rad} \Gamma, \operatorname{rad} \Gamma) \otimes_{O} O^{\prime} & \rightarrow \operatorname{Hom}_{\Gamma \otimes_{O} O^{\prime}}\left(\operatorname{rad} \Gamma \otimes_{O} O^{\prime}, \operatorname{rad} \Gamma \otimes_{O} O^{\prime}\right) \\
& =\operatorname{Hom}_{\Gamma^{\prime}}\left(\operatorname{rad} \Gamma^{\prime}, \operatorname{rad} \Gamma^{\prime}\right)
\end{aligned}
$$


from Lemma 4.1. The map

$$
\psi^{-1} \sigma(\phi \otimes 1): O_{l}(\operatorname{rad} \Gamma) \otimes_{O_{K}} O^{\prime} \rightarrow O_{l}\left(\operatorname{rad} \Gamma^{\prime}\right)
$$

is the identity, and the result is proved.

LEMMA 4.3. Let $\Lambda=\left(O_{L} / O_{K}, G, \rho\right)$ be a crossed product order in $A=(L / K, G, \rho)$ and suppose that $A$ splits over $K$. Then $\Lambda \cong$ $\left(O_{L} / O_{K}, G, 1\right)$.

Proof. Since the algebra $A$ is split over $K$, the class of $\rho$ in $H^{2}\left(G, L^{*}\right)$ is 1 . We shall show that the map $H^{2}\left(G, O_{L}^{*}\right) \rightarrow H^{2}\left(G, L^{*}\right)$ is one-to-one, and then the class of $\rho$ in $H^{2}\left(G, O_{L}^{*}\right)$ will be 1 , and the result will follow.

Let $E$ be the set of primitive idempotents of $L$ and let $M=\oplus_{\varepsilon \in E} Z \varepsilon$ be the free $Z$-module with basis $E$; $G$ acts on $M$ via its action on $E$. For $\varepsilon$ in $E$, let $v_{\varepsilon}$ be the normalized valuation on the field $L \varepsilon$, and define $v: L^{*} \rightarrow M$ by

$$
v(x)=\sum_{\varepsilon \in E} v_{\varepsilon}(x \varepsilon) \varepsilon, \quad x \in L^{*} .
$$

Then we get an exact sequence of $G$-modules

$$
o \rightarrow O_{L}^{*} \rightarrow L^{*} \stackrel{v}{\rightarrow} M \rightarrow o,
$$

giving rise to the exact sequence

$$
H^{1}(G, M) \rightarrow H^{2}\left(G, O_{L}^{*}\right) \rightarrow H^{2}\left(G, L^{*}\right) .
$$

Since $M$ is a permutation module, $M$ is isomorphic to the induced module Ind ${ }_{H}^{G}(\mathbf{Z})=\mathbf{Z} G \otimes_{Z H} \mathbf{Z}$, where $H$ is the stabilizer of an idempotent in $E$, and $H^{1}(G, M)=H^{1}(H, \mathbf{Z})=0$, since $H$ is finite. Then $H^{2}\left(G, O_{L}^{*}\right)$ $\rightarrow H^{2}\left(G, L^{*}\right)$ is one-to-one, as desired.

From Lemma 4.2, the chains

$$
\begin{aligned}
& \Lambda_{0} \subseteq \Lambda_{1} \subseteq \cdots \subseteq \Lambda_{s} \\
& \Lambda_{0}^{\prime} \subseteq \Lambda_{1}^{\prime} \subseteq \cdots \subseteq \Lambda_{s}^{\prime}
\end{aligned}
$$

have the same length, and $\Lambda_{s}^{\prime}$ is hereditary. Since the Theorem has been proved in the split case, and since $\Lambda^{\prime} \cong\left(O_{L^{\prime}} / O^{\prime}, G, 1\right)$, which follows from Lemma 4.3, we find that $s=d-(e-1)$. If $\Gamma$ is a hereditary order in $A$ containing $\Lambda$, then $\Gamma^{\prime}=\Gamma \otimes_{O_{K}} O^{\prime}$ is a hereditary order in $A^{\prime}$ containing $A^{\prime}$, and since $\Lambda_{s}^{\prime}$ is the unique minimal hereditary order in $A^{\prime}$ containing $A^{\prime}$, then $\Lambda_{s}^{\prime} \subseteq \Gamma^{\prime}$. We may embed $\Gamma$ in $\Gamma^{\prime}$ as $\Gamma \otimes_{O_{K}} 1$, and $A$ in $A^{\prime}$ as $A \otimes_{K} 1$, and then $\Gamma=\Gamma^{\prime} \cap A \supseteq \Lambda_{s}^{\prime} \cap A=\Lambda_{s}$, so $\Lambda_{s}$ is the unique minimal hereditary order in $A$ containing $\Lambda$. 
From [6, 39.14] we have

$$
\Lambda_{s} / \operatorname{rad} \Lambda_{s} \cong \prod_{i=1}^{r} M_{n_{t}}(\bar{\Delta})
$$

where $\bar{\Delta}=\Delta / \operatorname{rad} \Delta$, and $\Delta$ is the unique maximal order in $\operatorname{End}_{A}(V)$, with $V$ a simple $A$-module. Then

$$
\begin{aligned}
\Lambda_{s}^{\prime} / \operatorname{rad} \Lambda_{s}^{\prime} & \cong\left(\Lambda_{s} / \operatorname{rad} \Lambda_{s}\right) \otimes_{O_{K}} O^{\prime} \cong\left(\Lambda_{s} / \operatorname{rad} \Lambda_{s}\right) \otimes_{\bar{K}} \bar{K}^{\prime} \\
& \cong \prod_{i=1}^{r} M_{n_{i}}\left(\bar{\Delta} \otimes_{\bar{K}} \bar{K}^{\prime}\right) .
\end{aligned}
$$

Now $\bar{\Delta} \otimes_{\bar{K}} \bar{K}^{\prime} \cong\left(\bar{K}^{\prime}\right)^{m}$, where $m$ is the Schur index of $A$, since $\bar{K}$ is finite $([6,14.3])$. Thus

$$
\Lambda_{s}^{\prime} / \operatorname{rad} \Lambda_{s}^{\prime} \cong\left(\prod_{i=1}^{r} M_{n_{i}}\left(\bar{K}^{\prime}\right)\right)^{m} .
$$

Therefore the type number of $\Lambda_{s}^{\prime} / \operatorname{rad} \Lambda_{s}^{\prime}$, known to be $e$ from $\S 3$, is equal to $m r$, yielding

$$
r=\frac{e}{m}
$$

Each invariant $n_{l}=f$, since the invariants $n_{i}$ of $\Lambda_{s}^{\prime}$ are $f$. Therefore the proof of the theorem is complete.

5. Generators for $\Lambda_{s}$ in the split case. In this section we find generators for $\Lambda_{s}$ in the case that $\rho=1$. To simplify the exposition, we assume that $L$ is a field, which is totally ramified over $K$. We let $P_{L}$ be the maximal ideal of $O_{L}$, and let $v_{L}$ be the normalized valuation on $L$. Let $M_{i}$ denote the $\Lambda$-module $P_{L}^{i}, i \in \mathbf{Z}$.

LEMMA 5.1. Let $w=d-(e-1)$, and let $x$ be an element of $L$ such that $v_{L}(x)=-w$. Let $\alpha=x \sum_{g \in G} u_{g} \in A$. Then $\alpha M_{l} \subseteq M_{i}$, $i \in \mathbf{Z}$ (so $\alpha \in \Lambda_{s}$, from Lemma 3.2), and unless $i \equiv-w(\bmod e), \alpha M_{\imath} \subseteq M_{i+1}$, whereas if $i \equiv-w(\bmod e), \alpha M_{\imath} \nsubseteq M_{\imath+1}$.

Proof. Let $\operatorname{tr}$ denote the trace from $L$ to $K$. We first compute $\operatorname{tr}\left(P_{L}^{l}\right)$, $i \in \mathbf{Z}$. We have, for $j \in \mathbf{Z}$,

$$
\begin{aligned}
\operatorname{tr}\left(P_{L}^{i}\right) & \subseteq P_{K}^{j} \Leftrightarrow \operatorname{tr}\left(P_{L}^{i} P_{K}^{-j}\right) \subseteq O_{K} \\
& \Leftrightarrow \operatorname{tr}\left(P_{L}^{i-e j}\right) \subseteq O_{K} \Leftrightarrow P_{L}^{i-e j} \subseteq \mathscr{D}^{-1} \\
& \Leftrightarrow P_{L}^{i-e j+d} \subseteq O_{L} \Leftrightarrow i-e j+d \geq 0 \\
& \Leftrightarrow j \leq \frac{i+d}{e}
\end{aligned}
$$


(we have used $\mathscr{D}=P_{L}^{d}$ ). Thus

$$
\operatorname{tr}\left(P_{L}^{i}\right)=P_{K}^{[(i+d) / e]},
$$

where [ ] denotes greatest integer. Since $\sum u_{g} \cdot y=\sum g(y)=\operatorname{tr} y, y \in L$, we have

$$
O_{L} \alpha M_{i}=O_{L} x \operatorname{tr}\left(P_{L}^{i}\right)=x O_{L} P_{K}^{[(i+d) / e]}=x P_{L}^{e[(i+d) / e]} .
$$

Write

$$
\left[\frac{i+d}{e}\right]=\left[\frac{i+w}{e}+\frac{d-w}{e}\right]=\left[\frac{i+w}{e}+\frac{e-1}{e}\right] .
$$

If $(i+w) / e \notin \mathbf{Z}$, then $[(i+d) / e]>(i+w) / e$, so $e[(i+d) / e] \geq$ $i+w$, and

$$
O_{L} \alpha M_{i} \subseteq x P_{L}^{i+w+1}=P_{L}^{i+1}=M_{\imath+1} .
$$

If $(i+w) / e \in \mathbf{Z}$, then $[(i+d) / e]=(i+w) / e$, so $e[(i+d) / e]=i+$ $w$, and

$$
O_{L} \alpha M_{i}=x P_{L}^{++w}=M_{i} .
$$

This completes the proof.

Let $\pi_{L}$ be a prime element of $O_{L}$. Then from Lemma 3.2, we have $\pi_{L}^{-1} \Lambda_{s} \pi_{L}=\Lambda_{s}$. Let $\alpha=x \sum u_{g}$ be the element of Lemma 5.1, and define

$$
\alpha_{\imath}=\pi_{L}^{-i} \alpha \pi_{L}^{i}, \quad 0 \leq i<e .
$$

From Lemma 5.2, it follows that $\alpha_{i}$ acts non-trivially on $M_{-w+l} / M_{-w+i+1}$, whereas $\alpha_{i}$ annihilates $M_{j} / M_{j+1}$ if $j \neq-w+i(\bmod e)$. Thus the simple $\Lambda_{s}$-modules $M_{0} / M_{1}, M_{1} / M_{2}, \ldots, M_{e-1} / M_{e}$ are non-isomorphic, and hence form a complete set of simple $\Lambda_{s}$-modules. Recall that $\Lambda_{s} / \operatorname{rad} \Lambda_{s}$ $\cong \prod_{i=1}^{r} M_{n_{i}}(\bar{K})$, and each $n_{i}=f=1$, since we are assuming that $L / K$ is totally ramified. Hence $\Lambda_{s} / \operatorname{rad} \Lambda_{s}$ is commutative. Further, $r=e$, so $\operatorname{dim}_{\bar{K}}\left(\Lambda_{s} / \operatorname{rad} \Lambda_{s}\right)=e$. Then the elements $\alpha_{i}+\operatorname{rad} \Lambda_{s}$ generate $\Lambda_{s} / \operatorname{rad} \Lambda_{s}$ as a $\bar{K}$-module, $0 \leq i<e$. Since $\operatorname{rad} \Lambda_{s}=P_{L} \Lambda_{s}$, we see that $O_{L} \alpha_{i}, 0 \leq i$ $<e$, generate $\Lambda_{s}$ as an $O_{K}$ module. So $\pi_{i}^{\prime} \alpha_{i}, 0 \leq j<e, 0 \leq i<e$, generate $\Lambda_{s}$ as an $O_{K}$-module.

Finally, from the formula $\operatorname{tr}\left(P_{L}^{i}\right)=P_{K}^{[(i+d) / e]}$ from Lemma 5.1, if we set $i=-w$, then $i+d=e-1$, so $\operatorname{tr}\left(P_{L}^{-w}\right)=O_{K}$. Thus we may find $y$ in $L$ with $v_{L}(y)=-w$ such that $\operatorname{tr}(y)=u$ is a unit of $O_{K}$. Then $x=u^{-1} y$ has $v_{L}(x)=-w$ and $\operatorname{tr}(x)=1$. Now $\left(\sum u_{g}\right) x\left(\sum u_{g}\right)=\operatorname{tr}(x) \sum u_{g}=\sum u_{g}$, so $\alpha=x \sum u_{g}$ is idempotent. From the action of $\alpha$ on the simple modules $M_{i} / M_{i+1}$, we find that $\alpha$ is a primitive idempotent of $\Lambda_{s}$, and that the elements $\alpha_{i}+\operatorname{rad} \Lambda_{s}$ are all the primitive idempotents of $\Lambda_{s} / \operatorname{rad} \Lambda_{s}$. 
6. Complements. The results of Auslander-Goldman-Rim-Williamson mentioned in the Introduction follow easily from our Theorem. If $\rho=1, \Lambda$ is a maximal order in $A \Leftrightarrow s=0, r=1 \Leftrightarrow e / m=1 \Leftrightarrow e=1$, since $m=1$. For any $\rho, \Lambda$ is hereditary $\Leftrightarrow s=0 \Leftrightarrow d=e-1 \Leftrightarrow L / K$ is tamely ramified, from [7, Prop. 13, p. 67].

We also recover a result of Janusz [4], who showed that, in the tamely ramified case, $\Lambda$ has type $e / m$ and invariants $f$. (See also Merklen [5].)

From the fact that $r=e / m$, we find a way to compute the Schur index $m$ of $A$ as follows: the centre of $\Lambda_{s} / \operatorname{rad} \Lambda_{s}$ has $e / m$ component fields (each of dimension $m$ over $\bar{K}$ ).

It may be shown that the index

$$
\left(\Lambda_{s}: \Lambda\right)=\pi_{K}^{n^{2}(d-(e-1)) / 2 e}
$$

where $n=[L: K]$. This follows from

$$
(\tilde{\Lambda}: \Lambda)=\left(\tilde{\Lambda}_{s}: \Lambda_{s}\right)\left(\Lambda_{s}: \Lambda\right)^{2} .
$$

Note that Lemma 3.4 (that $\tilde{\Lambda}=P_{K}^{-1} \operatorname{rad} \Lambda$ if $\Lambda$ is hereditary) also holds in the non-split case, as may be shown by tensoring with an unramified extension.

In the split case (§3), the $\Lambda$-lattices contained in a irreducible $A$-module $V$ are linearly ordered, but this fails to be true if $A$ is not split. However, it may be shown, in general, that the $\Lambda$-lattices $M$ in $V$ such that $\operatorname{End}_{\Lambda}(M)$ is the maximal order in $\operatorname{End}_{A}(V)$ are linearly ordered, and this can be used to prove the Theorem, just as in $\S 3$.

Note that we could have used right-orders $\Lambda_{j+1}^{\prime}=O_{r}\left(\operatorname{rad} \Lambda_{j}^{\prime}\right)$ throughout, instead of left orders, and still obtain the same answer $s=d-(e-1)$ for the length of the chain $\Lambda_{0}^{\prime} \subseteq \cdots \subseteq \Lambda_{s}^{\prime}$. By uniqueness of $\Lambda_{s}$, we would get $\Lambda_{s}=\Lambda_{s}^{\prime}$, but we do not know whether $\Lambda_{j}=\Lambda_{j}^{\prime}$ for all $j, 1<j<s$.

\section{REFERENCES}

[1] M. Auslander and O. Goldman, Maximal orders, Trans. Amer. Math. Soc., 97 (1960), $1-24$.

[2] M. Auslander and D. S. Rim, Ramification index and multiplicity, Illinois J. Math., 7 (1963), 566-581.

[3] H. Benz and H. Zassenhaus, Über verschränkte Produktordnungen, J. Number Theory, 20 (1985), 282-298.

[4] G. Janusz, Crossed product orders and the Schur index, Comm. Algebra, 8 (1980), 697-706. 
[5] H. Merklen, Hereditary crossed product orders, Pacific J. Math., 74 (1978), 391-406.

[6] I. Reiner, Maximal Orders, Academic Press, London, 1975.

[7] J.-P. Serre, Corps Locaux, Act. Sci. et Ind. 1296, Hermann, Paris, 1962.

[8] S. Williamson, Crossed products and hereditary orders, Nagoya Math. J., 23 (1963), $103-120$.

Received September 21, 1984 and in revised form March 26, 1985. Supported by NSERC.

UNIVERSITy OF Alberta

EDMOnTon, Alberta

Canada T6G 2G1 



\section{PACIFIC JOURNAL OF MATHEMATICS EDITORS}

\author{
V. S. VARAdarajan (Managing Editor) \\ University of California \\ Los Angeles, CA 90024 \\ Hebert Clemens \\ University of Utah \\ Salt Lake City, UT 84112 \\ Charles R. DePrima \\ California Institute of Technology \\ Pasadena, CA 91125
}

R. FINN

Stanford University

Stanford, CA 94305

HeRManN FLASChKa

University of Arizona

Tucson, AZ 85721

RAMESH A. GANGOlli

University of Washington

Seattle, WA 98195

ROBION KIRBY

University of California

Berkeley, CA 94720

\author{
C. C. MOORE \\ University of California \\ Berkeley, CA 94720 \\ H. SAMELSON \\ Stanford University \\ Stanford, CA 94305 \\ HAROLD STARK \\ University of California, San Diego \\ La Jolla, CA 92093
}

\section{ASSOCIATE EDITORS}

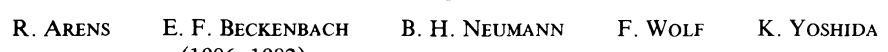

(1906-1982)

\section{SUPPORTING INSTITUTIONS}

\begin{abstract}
UNIVERSITY OF ARIZONA
UNIVERSITY OF BRITISH COLUMBIA

CALIFORNIA INSTITUTE OF TECHNOLOGY

UNIVERSITY OF CALIFORNIA

MONTANA STATE UNIVERSITY

UNIVERSITY OF NEVADA, RENO

NEW MEXICO STATE UNIVERSITY

OREGON STATE UNIVERSITY
\end{abstract}

\author{
UNIVERSITY OF OREGON \\ UNIVERSITY OF SOUTHERN CALIFORNIA \\ STANFORD UNIVERSITY \\ UNIVERSITY OF HAWAII \\ UNIVERSITY OF TOKYO \\ UNIVERSITY OF UTAH \\ WASHINGTON STATE UNIVERSITY \\ UNIVERSITY OF WASHINGTON
}

The Supporting Institutions listed above contribute to the cost of publication of this Journal, but they are not owners or publishers and have no responsibility for its content or policies.

Mathematical papers intended for publication in the Pacific Journal of Mathematics should be in typed form or offset-reproduced (not dittoed), double spaced with large margins. Please do not use built up fractions in the text of the manuscript. However, you may use them in the displayed equations. Underline Greek letters in red, German in green, and script in blue. The first paragraph must be capable of being used separately as a synopsis of the entire paper. In particular it should contain no bibliographic references. Please propose a heading for the odd numbered pages of less than 35 characters. Manuscripts, in triplicate, may be sent to any one of the editors. Please classify according to the scheme of Math. Reviews, Index to Vol. 39. Supply name and address of author to whom proofs should be sent. All other communications should be addressed to the managing editor, or Elaine Barth, University of California, Los Angeles, California 90024.

There are page-charges associated with articles appearing in the Pacific Journal of Mathematics. These charges are expected to be paid by the author's University, Government Agency or Company. If the author or authors do not have access to such Institutional support these charges are waived. Single authors will receive 50 free reprints; joint authors will receive a total of 100 free reprints. Additional copies may be obtained at cost in multiples of 50 .

The Pacific Journal of Mathematics is issued monthly as of January 1966. Regular subscription rate: $\$ 190.00$ a year (5 Vols., 10 issues). Special rate: $\$ 95.00$ a year to individual members of supporting institutions.

Subscriptions, orders for numbers issued in the last three calendar years, and changes of address should be sent to Pacific Journal of Mathematics, P.O. Box 969, Carmel Valley, CA 93924, U.S.A. Old back numbers obtainable from Kraus Periodicals Co., Route 100, Millwood, NY 10546.

The Pacific Journal of Mathematics at P.O. Box 969, Carmel Valley, CA 93924 (ISSN 0030-8730) publishes 5 volumes per year. Application to mail at Second-class postage rates is pending at Carmel Valley, California, and additional mailing offices. Postmaster: Send address changes to Pacific Journal of Mathematics, P.O. Box 969, Carmel Valley, CA 93924.

PUBLISHED BY PACIFIC JOURNAL OF MATHEMATICS, A NON-PROFIT CORPORATION

Copyright $\odot 1986$ by Pacific Journal of Mathematics 


\section{Pacific Journal of Mathematics}

\section{Vol. 122, No. 2 \\ February, 1986}

Gideon Amit and David Chillag, On a question of Feit concerning character values of finite solvable groups ......................257

Constantin Gelu Apostol and Frank Larkin Gilfeather, Isomorphisms modulo the compact operators of nest algebras ................263

Parviz Azimi and James Neil Hagler, Examples of hereditarily $l^{1}$ Banach

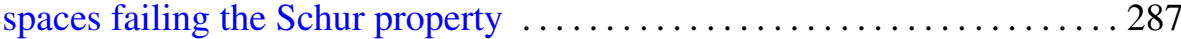

Brian Evan Blank, Boundary behavior of limits of discrete series representations of real rank one semisimple groups . . . . . . . . . . 299

Jeffrey Carroll, Some undecidability results for lattices in recursion theory

Gerald Howard Cliff and Alfred Rheinhold Weiss, Crossed product and

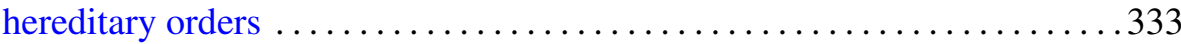

Ralph Cohen, Realizing transfer maps for ramified coverings . . . . . . . . 347

Ronald James Evans, Hermite character sums . .................. 357

C. L. Frenzen and Roderick Sue-Chuen Wong, Asymptotic expansions of the Lebesgue constants for Jacobi series . . . . . . . . . . . . . . . . 391

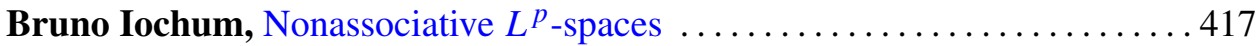

John McDonald, Unimodular approximation in function algebras ....... 435

John Robert Quine, Jr., Ramification and unintegrated value distribution . . 441

Marc Raphael, Commutants of quasisimilar subnormal operators ........ 449

Parameswaran Sankaran and Peter Zvengrowski, On stable

parallelizability of flag manifolds

Helga Schirmer, A relative Nielsen number

Barry Simon, Schrödinger semigroups on the scale of Sobolev spaces . . . . . 475

Viakalathur Shankar Sunder, Stochastic integration in Fock space

Jan de Vries, A note on the $G$-space version of Glicksberg's theorem 